\title{
Silicon impact on carbon ordering at the martensite lattice: molecular dynamics simulations
}

\author{
P. V. Chirkov ${ }^{\dagger}$ A. A. Mirzoev, D. A. Mirzaev \\ †p.chirkow@gmail.com
}

\author{
South Ural State University, Lenina Prospekt, 76, Chelyabinsk, 454080, Russia
}

\begin{abstract}
Despite their widespread use in the industry, silicon-alloyed bainitic steels remain insufficiently studied in many respects. For instance, there is no available information on the impact of silicon impurities on the properties of martensitic structure. The present article deals with this issue. The results of a computer simulation of the impact of silicon impurities on the tetragonal distortion of martensite lattice and the interaction of carbon atoms in a body-centered cubic (bcc) lattice of iron using the molecular dynamics method are presented. Interatomic potentials that make it possible to describe the interactions of Fe-Si-C in martensite within the framework of the embedded atom model (EAM) are developed. It has been established that when silicon is added to steel, the lattice constant $c$ decreases noticeably and the constant $a$ increases slightly. The tetragonality expressed by $c / a$ ratio decreases with respect to the results of Kurdyumov's experiment for any carbon concentrations. The impact of silicon on the formation of martensite was studied by minimizing the energy of the strain-induced interaction parameter $\lambda_{2}(0)$ from the order-disorder transition theory in Zener-Khachaturyan interstitial solutions, which determines the critical temperature of the bcc-bct (body-centered tetragonal) transition. The calculations do not confirm the direct proportional dependence of the change in the tetragonality of martensite and the nature of carbon activity variation during alloying with silicon, which increases activity but decreases tetragonality.
\end{abstract}

Keywords: martensitic transformation, silicon, ordering, molecular dynamics.

\section{Introduction}

Recently, due to a new generation of nanostructured bainitic steels, a unique combination of strength $\left(\sigma_{T} \approx 2.1 \mathrm{GPa}\right)$, viscosity $\left(K_{I C} \approx 34 \mathrm{MPa} \cdot \mathrm{m}^{1 / 2}\right)$, and plasticity $(\delta \approx 7 \%)$ has been achieved. To obtain a similar level of these properties, it is necessary to suppress the release of carbides during the formation of bainite. For that purpose, G. Bhadeshia $[1,2]$ proposed the introduction silicon into steel (up to $2 \mathrm{wt} . \%$ ), and $[3,4]$ showed the practical effect of such introduction. As a result, interest in alloying of steels with silicon rose again.

The Fe-Si system, the properties of which significantly determine the characteristics of silicon-alloyed steels, was studied in detail experimentally. The phase diagram was presented in [5], wherein up to $\sim 10$ at. $\%$, the $\alpha$-phase, which is a disordered solution of silicon in the $b c c$ iron, is stable. With the growing concentration of silicon to 6 at.\%, there is a weak $0.22 \%$ decrease of the bcc lattice constant [6]. With a subsequent increase in the silicon content, the system will be a mixture of an $\alpha_{1}$-phase $\left(\mathrm{Fe}_{3} \mathrm{Si}\right)$ with a $\mathrm{D}_{3}$ lattice and an $\alpha_{2}$-phase $(\mathrm{FeSi})$ with a B2 structure. Usually, steels contain less than 6 at.\% (or 3 wt.\%) of Si.

In [7], L. Yu. Kremnev proposed a new sight on the impact of alloying elements on the martensite tetragonality. He came to a conclusion that the tetragonality $c / a$ of martensite steels depended not only on the carbon concentration but also on the content of alloying elements therein and the specific features of their interaction with carbon atoms. He considered the intensity of variation in the carbon activity coefficient in steel under the influence of an alloying element as a characteristic of such interaction. The presence of strong carbide-forming components in steel, which reduce the coefficient of carbon activity, leads to a significant decrease of tetragonality. In contrast, the presence of non-carbideforming elements in the martensite lattice, which increase the coefficient of carbon activity, causes the formation of an anomalously large tetragonality of martensite. The last group of elements includes $\mathrm{Al}, \mathrm{Ni}$, and $\mathrm{Si}$. As for nickel and aluminum, which addition can actually cause abnormal high tetragonality [8], we agree with Kremnev's view. When silicon is introduced into steel, the activity of carbon also increases and partial factors of carbon activity for ferrite and austenite are expressed by the formulae:

$$
\ln f_{\mathrm{C}}^{\mathrm{Si}}=10.35 N_{\mathrm{Si}}, \quad \ln f_{\mathrm{C}}^{\mathrm{Si}}=14.5 N_{\mathrm{Si}},
$$

where $N_{\mathrm{Si}}$ is the atomic fraction of silicon in the alloy, and do not depend on carbon content [9]. The authors noted that the independence of $f_{\mathrm{C}}^{\mathrm{Si}}$ from temperature, both in ferrite and in austenite, indicates that the impact of silicon on carbon activity are expressed by a change of the partial vibrational entropy of carbon, rather than its partial energy. At the same time, the impact of silicon on the activity of carbon in ferrite is stronger than in austenite. It should also be noted that silicon addition increases the activity of carbon more strongly. Consequently, according to Kremnev's theory, silicon should increase the tetragonality of martensite.

Since martensitic transformation is diffusionless and is produced by shear displacement of atoms to a distance 
less than interatomic, martensite will inherit the mutual arrangement of the carbon atoms and the alloying element that exists in austenite near the martensitic point. Therefore, the effects of activity in austenite should be considered in Kremnev's concept. However, no experimental studies for the $\mathrm{Fe}-\mathrm{Si}-\mathrm{C}$ system have been found in the literature.

In [10], the interaction of carbon and silicon atoms located at different distances in the crystal lattice of iron was calculated. It was found that in the first two coordination shells, a repulsive potential of 0.48 and $0.75 \mathrm{eV}$, respectively, influences the interstitial impurity, and at large distances the $\mathrm{Si}-\mathrm{C}$ interaction drops to zero. Based on this data, it would be logical to consider the impact of silicon impurities on the ordering of carbon atoms in martensite. Therefore, we propose computer simulation of this system by the molecular dynamics method, using EAM-type interatomic potentials, which ensure a fairly accurate reproduction of the Fe-Si-C system properties.

\section{Fitting of interatomic potential in the $\mathrm{Fe}-\mathrm{Si}-\mathrm{C}$ martensite}

Realistic interaction between atoms can be obtained only when the electronic subsystem is considered using the electron density functional theory. However, this method requires huge computing resources, and its capabilities are very limited (no more than 200 ions in a time interval of about $100 \mathrm{ps}$ ).

The number of particles under consideration can be increased using the method of classical molecular dynamics based on Embedded Atom Method (EAM) interatomic potentials. These potentials, developed by D. Daw and M. Baskes [11] and reformulated by Finnis and Sinclair [12], give sufficiently realistic calculation results when appropriate fitting based on the results of the first-principles simulation has been made. In the EAM, the potential energy of the $i$-th atom of type $\alpha$ has the form:

$$
U_{\alpha, i}=1 / 2 \sum_{j \neq 1} \phi_{\beta \alpha}\left(r_{i j}\right)+F_{\alpha}\left(\rho_{i}\right),
$$

where the first term is a pair interaction of ions and the second term is an interaction of the ion with the remaining system by embedding energy $F(\rho)$, which represents the difference between the energies of an atom embedded in the electron gas and an isolated atom. The dependence of energy on the total electron density characterizes the interaction of an ion with an electronic system, wherein the total density created at a distance $r$ from the $i$-th ion is equal to the sum:

$$
\rho_{i}=\sum_{j \neq i} \psi_{\beta \alpha}\left(r_{i j}\right)
$$

where $\psi(r)$ represents electron density created by the atom $j$ of type $\beta$ in the point of the atom $i$ of type $\alpha$.

We propose the following model for building interatomic interaction potentials in ternary dilute alloys of the Fe-Si-C system with a $b c c$ lattice. The basis used is the EAM-type potential for the binary $\mathrm{Fe}-\mathrm{C}$ system [13] which, as was shown earlier [14], allows us to adequately describe the interaction of carbon atoms in $b c c$ iron, which leads to the appearance of a tetragonal martensite lattice. According to $a b$ initio calculations carried out in [15], the substituted Si atoms do not significantly distort the $\alpha$-iron crystal lattice and do not cause changes in the short-range order. In addition, there is strong repulsion between silicon and carbon atoms in the first two coordination spheres. These results allow us to assume that the $\mathrm{Si}-\mathrm{Fe}$ and $\mathrm{Si}-\mathrm{Si}$ interactions do not differ too much from the Fe-Fe potential. Therefore, we assume that for the potentials determined by Eq. (1) the equality of the corresponding functions would be fair: $F_{\mathrm{Si}}=F_{\mathrm{Fe}}, \phi_{\mathrm{FeSi}}=\phi_{\mathrm{FeFe}}$ ' and $\psi_{\mathrm{FeSi}}=\psi_{\mathrm{SiFe}}=\psi_{\mathrm{FeFe}}$. As the pairs of Si-C atoms cannot approach each other to the distance of overlapping electron orbitals, their interactions (1) preserved only the pair part of the potential, turning the corresponding functions of electron densities to zero: $\psi_{\mathrm{CSi}}=\psi_{\mathrm{SiC}}=0$.

The Si-C interaction was fitted to the data of the firstprinciples simulation [10], wherein the pair potential approximation was considered. Therefore, the corresponding electron density functions were set to zero: $\psi_{\mathrm{CSi}}=\psi_{\mathrm{SiC}}=0$. The pair interaction $\phi_{\mathrm{Sic}}(r)$, which is a series of splines with continuous first-order and second-order derivatives, had a functional form as introduced in [16] and was optimized for the values of the binding energy of silicon with carbon $E_{\mathrm{SiC}}^{b}(\rho)$, which is calculated as:

$$
\begin{aligned}
E_{\mathrm{SiC}}^{b}(\rho)= & {\left[E_{\rho}((N-1) \mathrm{Fe}+\mathrm{Si}+\mathrm{C})-E(N \mathrm{Fe}+\mathrm{C})\right]-} \\
& -[E((N-1) \mathrm{Fe}+\mathrm{Si})-E(N \mathrm{Fe})],
\end{aligned}
$$

where $E(N \mathrm{Fe})$ is the energy of a perfect lattice containing $N$ atoms of iron; $E((N-1) \mathrm{Fe}+\mathrm{Si})$ and $E(N \mathrm{Fe}+\mathrm{C})$ represent the energies of the systems containing the silicon replacement atom and the carbon atom embedded in the octahedral site of the lattice, respectively. The energy of the supercell with atoms of silicon and carbon located at the distance $\rho$ is denoted by $E_{\rho}((N-1) \mathrm{Fe}+\mathrm{Si}+\mathrm{C})$. With this calculation method, positive values of $E_{\mathrm{Sic}}^{b}(\rho)$ correspond to repulsion between silicon and carbon impurities, and negative values correspond to attraction.

It can be seen from Table. 1 that the potential has been optimized rather accurately for the available results of the first-principles simulation of the $\mathrm{Si}-\mathrm{C}$ interaction in the iron lattice. According to the data from [10], repulsion between $\mathrm{Si}$ and $\mathrm{C}$ atoms reaches its maximum value of $0.8 \mathrm{eV}$ in the second coordination sphere at the distance $\sqrt{2} a_{0} / 2$, where $a_{0}$ is the iron lattice constant, and it becomes practically equal to zero when placed in the third coordination shell. Fig. 1 shows the form of an optimized pair interaction function $\phi_{\mathrm{SiC}}(r)$. The position of the local minimums corresponds to the first two configurations.

\section{Computer simulations}

Diffusion of impurities is important in many process especially the carbon diffusion in steels. Alloying substituted elements can make strong influence on interstitials mobility. Molecular dynamics simulations are the powerful method to predict many thermally activated process. According to the theory of random walks, diffusion coefficient $D$ is express as:

$$
D=\frac{1}{6} \frac{\left\langle r^{2}\right\rangle}{t},
$$

where $\left\langle r^{2}\right\rangle$ is the mean square displacement of carbon atoms over time t.

We used LAMMPS package [17] to calculate the diffusion 
coefficient of carbon atoms in ferrite. The simulation cell consisted of $30 \times 30 \times 30$ unit cell translations $(54,000$ atoms). Temperature and pressure was controlled by the Nose-Hover $[18,19]$ thermostat and barostat. Periodical boundary conditions were applied along all directions in our simulations. In pure $b c c \mathrm{Fe}$, the diffusion coefficient of carbon atoms equals $1.53 \cdot 10^{-10} \mathrm{~m}^{2} / \mathrm{s}$, it has sufficiently great error compared to results of the Monte-Carlo simulations presented in [10], where $D=9 \cdot 10^{-12} \mathrm{~m}^{2} / \mathrm{s}$, but experiments do not result in such difference: $1.39 \cdot 10^{-11}[20], 8.04 \cdot 10^{-11} \mathrm{~m}^{2} / \mathrm{s}$ [21].

Carbon diffusivity is reduced by silicon content as is shown in Fig. 2 where the normalized diffusion coefficient is plotted versus silicon concentration. For instance, 8 at.\% $\mathrm{Si}$ reduces the diffusion coefficient with $\sim 45 \%$ at $1000 \mathrm{~K}$. $\mathrm{C}$ diffusivity is decreased because the first- and secondneighbor shells around $\mathrm{Si}$ are so high in energy that $\mathrm{C}$ is blocked from these sites. Qualitatively agreement between the $\mathrm{MD}$ results for fitted potential and Monte-Carlo simulations is evident.

Iron atoms in the $b c c$ lattice form irregular octahedrons, in which one space diagonal is shorter than the other two. When the carbon atom enters the octahedral sites of $\alpha$-iron,

Table 1. The binding energy of $\mathrm{Si}-\mathrm{C}$ in the $\alpha$-iron lattice for various configurations. The position of the interstitial carbon atom is indicated in the table, and the substituted silicon has the coordinates [000]. The results for the fitted potential built and available data of the first-principles calculations that were used in the optimization are presented here.

\begin{tabular}{|c|c|c|c|}
\hline$\#$ & C coordinates & DFT [10], eV & EAM, eV \\
\hline 1 & {$[1 / 200]$} & 0.478 & 0.54 \\
\hline 2 & {$[1 / 21 / 20]$} & 0.75 & 0.8 \\
\hline 3 & {$\left[1 \frac{1}{2} 0\right]$} & -0.021 & 0.02 \\
\hline 4 & {$\left[1 \frac{1}{2} 1 / 2\right]$} & -0.057 & 0 \\
\hline
\end{tabular}

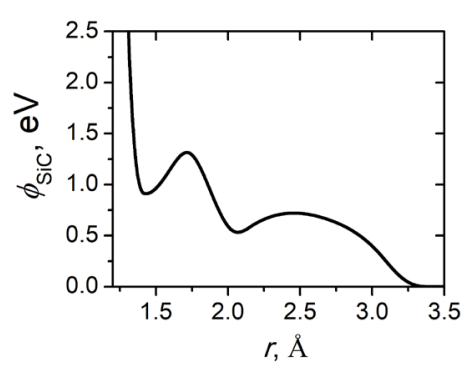

Fig. 1. Pair potential of $\mathrm{Si}-\mathrm{C}$ interaction.

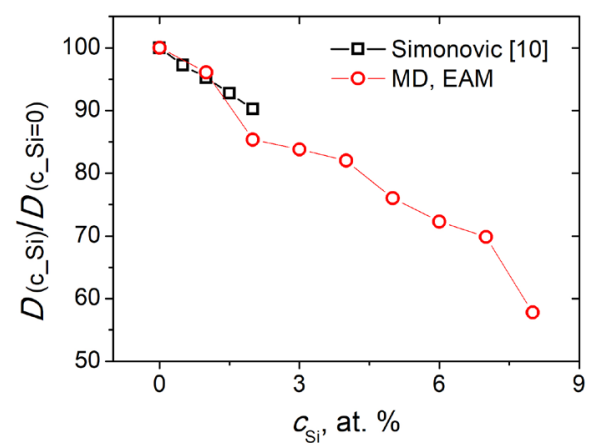

Fig. 2. Calculated diffusion coefficient of carbon in the presence of silicon substitutes relative to diffusion without $\mathrm{Si}$, in $b c c$ Fe when temperature equals to $1000 \mathrm{~K}$. the lattice is stretched only along one of the cube's edges. In the $b c c$ lattice, there is an equal number of octahedral sites, in which the direction of the short diagonal is oriented along the $x, y$, and $z$ axes. Accordingly, it is necessary to distinguish $x, y$, and $z$ site types. When the carbon atom is in pore $z$, it stretches the lattice locally in the direction of axis $z$.

According to the Zener-Khachaturyan theory [22, 23], the properties of iron based martensite, for example, lattice constants and tetragonal distortion, depend on the distribution of carbon atoms in octapores of different sublattices, and this dependence is determined by the magnitude of the strain induced interaction parameter $\lambda_{2}(0)$. However, the impact of silicon impurities on the value of this parameter is still unstudied.

We showed previously in [14] that the value of the parameter $\lambda_{2}(0)$ within the framework of atomistic modeling can be determined using the expression for the free energy of martensite with the long range order parameter $\eta$ from the theory of K. Zener and A. G. Khachaturian [23]:

$\begin{aligned} F\left(c_{\mathrm{C}}, \eta\right) & =F\left(c_{\mathrm{C}}, 0\right)-1 / 3 N \lambda_{2}(0) \cdot c_{\mathrm{C}}^{2} \eta^{2}+ \\ & +2 / 3 N c_{\mathrm{C}} k T[(1-\eta) \ln (1-\eta)+(1+2 \eta) \ln (1+2 \eta)],\end{aligned}$

where $N$ is the number of atoms in the crystal lattice and $c_{C}-$ the content of carbon atoms from this number. The long range order parameter is determined by the following expression:

$$
\eta=\frac{n_{\mathrm{C}}^{z}-1 / 3 n_{\mathrm{C}}}{2 / 3 n_{\mathrm{C}}}
$$

This shows an excess of carbon atoms in $z$-octahedral sites compared to the amount that should be present when $x$-, $y$-, and $z$-sites $\left(n_{\mathrm{C}}^{x}=n_{\mathrm{C}}^{y}=n_{\mathrm{C}}^{z}=n_{\mathrm{C}} / 3\right)$ are uniformly filled with carbon atoms. As can be seen from the definition, the long range order parameter can vary from zero (disordered state) to 1 (ordered state).

If martensite is fully ordered, then

$$
F\left(c_{\mathrm{C}}, 1\right)=F\left(c_{\mathrm{C}}, 0\right)+E_{z}+k_{B} T N c_{\mathrm{C}} \ln 3,
$$

where $E_{z}=-N \cdot \lambda_{2}(0) \cdot c_{C}^{2} / 3$ represents the energy of the strain induced interaction of carbon atoms if they are in an ordered state, i.e. all carbon atoms are in the $z$ sublattice, $\eta=1$. In turn,

$$
F\left(c_{\mathrm{C}}, 0\right)=E_{x y z}-k_{B} T \cdot N \cdot c_{\mathrm{C}} \cdot \ln 3,
$$

where $E_{x y z}$ is the energy of interaction of carbon atoms in disordered ferrite. Based on these expressions, we obtained the formula:

$$
\lambda_{2}(0)=3 \cdot \frac{E_{x y z}-E_{z}}{N c_{\mathrm{C}}^{2}},
$$

which was further used to calculate the $\lambda_{2}(0)$ parameter.

The energies $E_{x y z}$ and $E_{z}$ were calculated by minimizing the energy of the supercell, which is a $b c c$ lattice of iron with the dimensions of $30 \times 30 \times 30$ of elementary cells (54,000 atoms). Silicon and carbon atoms were randomly distributed among the crystal lattice and octahedral interstices, respectively. The energies $E_{x y z}$ and $E_{z}$ were averaged by 100 configurations; carbon content of 4 at.\% was chosen. It should be noted that, according to Zener-Khachaturian theory, the strain-induced interaction parameter does not depend on the carbon content. As silicon concentration increases, the parameter $\lambda_{2}(0)$ decreases (Fig. 3) up to $4.2 \mathrm{eV} /$ atom (-18\%) at 10 at.\% 
of $\mathrm{Si}$, which is probably preconditioned by a strong repulsion of silicon and carbon atoms in close coordination shells.

Fig. 4 shows the concentration dependence of the lattice constants $c$ and $a$ of the martensite for $\mathrm{Fe}-\mathrm{C}$ and $\mathrm{Fe}-\mathrm{C}-\mathrm{Si}$ solutions with 10 at.\% of Si compared to experimental results from Kurgjumov [24]. It can be seen that the introduction of silicon reduces the lattice constants and, correspondingly, the tetragonal distortion $(c / a)$ of $\mathrm{Fe}-\mathrm{Si}-\mathrm{C}$ martensite lattice.

\section{Conclusions}

In this paper, we proposed EAM-type interatomic potentials for the Fe-Si-C system which correctly reproduce the data of the first-principles simulation of the interaction energy between silicon and carbon atoms located at different distances in the $\alpha$-iron lattice. The dependence of $\mathrm{C}$ diffusion on silicon content also qualitatively agrees with Mote-Carlo simulations at $0-2$ at. $\% \mathrm{Si}$.

Simulation of the tetragonality of martensite of siliceous steels carried out by molecular dynamics method made it possible to establish that the addition of silicon significantly reduces the constant $c$ and slightly increases the constant $a$. The $c / a$ ratio decreases relative to Kurdyumov's experiment results at all carbon content level.

Using the Zener-Khachaturyan theory, we calculated the dependence of the strain-induced interaction parameter in the Fe-C-Si triple solution on the silicon content. An increase in the concentration of silicon within the range up to 10 at.\% leads to monotonic decrease in the parameter $\lambda_{2}(0)$ from 5.2 to $4.2 \mathrm{eV} /$ atom.

Thus, it must be admitted that Kremnev's concept [7] of the direct proportional dependence of change in the tetragonality of martensite and the nature of carbon activity variation during the alloying of steels with different elements is not confirmed with respect to silicon. Silicon increases the activity of carbon in steel, but reduces tetragonality.

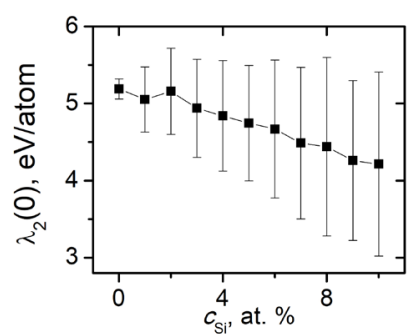

Fig. 3. Dependence of strain induced interaction parameter on the silicon concentration.

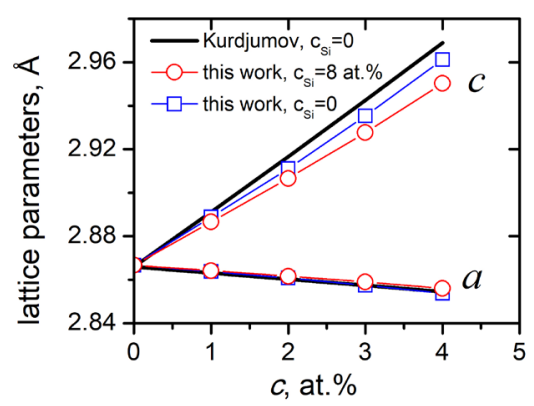

Fig. 4. Dependences of the lattice parameters on the carbon concentration. G.V. Kurdjumov's experimental results and data obtained from a simulation using EAM potentials.
Acknowledgements The work was supported by Act 211 Government of the Russian Federation, contract № 02 . A03.21.0011.

\section{References}

1. H.K. D. H. Bhadeshia, D. V. Edmonds. Metall. Mater. Trans. A 10, 895 (1979). DOI: 10.1007/BF02658309.

2. H. K. D. H. Bhadeshia, D. V. Edmonds. Metal Sci. 17, 411 (1983). DOI: 10.1179/030634583790420600.

3. C. Garcia-Mateo, F.G. Caballero, H.K. D. H. Bhadeshia. ISIJ Int. 43, 1238 (2003). DOI: 10.2355/ isijinternational.43.1238.

4. C. Garcia-Mateo, F. G. Caballero. ISIJ Int. 45, 1736 (2005). DOI: 10.2355/isijinternational.45.1736.

5. B. Predel. Fe-Si (iron-silicon) in Dy-Er-Fr-Mo (Springer, Berlin, 1995). DOI: 10.1007/10474837_1.

6. F. Richter, W. Pepperhoff. Steel Res. Int. 45, 107 (1974).

7. L. S. Kremnev, Tech. Phys. 83, 47 (2013). DOI: 10.1134/ S106378421309017X.

8. M.L. Bernstein, L.M. Kaputkina, S.D. Prokoshin. Steel tempering. Moscow, MISIS, 368 p. (in Russian) [М. Л. Бернштейн, Л. М. Капуткина, С. Д. Прокошкин. Отпуск стали. Москва, МИСИС, 1997, 368 с.]

9. B.M. Mogutnov, I.A. Tomilin, L.A. Shwartzman. Thermodynamics of iron-carbon alloys. Moscow, Metallurgia, 1972, 328 p. (in Russian) [Б. М. Могутнов, И. А. Томилин, Л. А. Шварцман. Термодинамика железо-углеродистых сплавов. Москва, Металлургия, 1972,328 c.]

10. D. Simonovic, C. K. Ande, A. I. Duff et al. Phys. Rev. B. 81, 054116 (2010). DOI: 10.1103/PhysRevB.81.054116.

11. M. S. Daw, S. M. Foiles, M. I. Baskes. Mat. Sci. Rep. 9, 251 (1993). DOI: 10.1016/0920-2307(93)90001-U.

12. M.W. Finnis, J.E. Sinclair. Phil. Mag. A. 50, 45 (1984). DOI: 10.1080/01418618408244210

13. T. Lau, C. J. F. Forst, X. Lin et al. Phys. Rev. Lett. 98, 215501 (2007). DOI: 10.1103/PhysRevLett.98.215501.

14. P. V. Chirkov, A.A. Mirzoev, D.A. Mirzaev. The Phys. Met. Metall. 117, 1 (2016). DOI: 10.1134/S0031.

15. E. Vincent, C.S. Becquart, C. Domain. Nucl. Instr. Meth. Phys. Res. B. 228, 137 (2005). DOI: 10.1016/j. nimb.2004.10.035.

16. M. I. Mendelev, S. Han, D. J. Srolovitz et al. Phil. Mag. 83, 3977 (2003). DOI: 10.1080/14786430310001613264.

17. S. Plimton. J. Comp. Phys. 117, 1 (1995). DOI: 10.1006/ jcph.1995.1039.

18. W. G. Hoover. Phys. Rev. A. 31, 1695 (1985). DOI: 10.1103/PhysRevA.31.1695.

19. S. Nose. J. Phys.: Cond. Matter. 2, 115 (1990). DOI: 10.1088/0953-8984/2/S/013.

20. R. B. McLellan, M.L. Rudee, T. Ishibachi. AIME Met. Soc. Trans. 233, 1938 (1965).

21. E. A. Brandes. Smithells Metals Reference Book. London, Butterworths, 1983, $1025 \mathrm{p}$.

22. C. Zener. Phys. Rev. 74, 639 (1948). DOI: 10.1103/ PhysRev.74.639.

23. A. G. Khachaturyan. Theory of Structural Transformations in Solids. New York, Wiley, 1983, 353 p.

24. G. V. Kurdjumov, E. S. Kaminsky. Nature. 122, 475 (1928). 